\title{
3'-O-Acetyl-24-Epi-7,8-Didehydrocimigenol-3-O- $\beta$-D- Xylopryranoside Decreases Amyloid Beta Production in Amyloid Precursor Protein-Transfected HeLa Cells
}

\author{
Sang-Bin Lee ${ }^{1,2, \dagger}$, Ansun Park ${ }^{2,3, \dagger}$, Chi Thanh Ma ${ }^{4}$, Young Ho Kim ${ }^{5}$ and Hyun Ok Yang ${ }^{1,2,3, *}$ \\ ${ }^{1}$ Department of Integrative Biological Sciences and Industry, Sejong University, Seoul 05006, \\ ${ }^{2}$ Natural Product Research Center, Korea Institute of Science and Technology, Gangneung 25451, \\ ${ }^{3}$ Division of Bio-Medical Science \& Technology, KIST School, Korea University of Science and Technology, Seoul 02792, Republic of \\ Korea \\ ${ }^{4}$ Department of Pharmacognosy, University of Medicine and Pharmacy at Ho Chi Minh City, Ho Chi Minh City 700000, Vietnam \\ ${ }^{5}$ College of Pharmacy, Chungnam National University, Daejeon 34134, Republic of Korea
}

\begin{abstract}
Extracellular beta amyloid $(A \beta)$ plaques are the neuropathological hallmarks of Alzheimer's disease (AD). Accordingly, reducing $A \beta$ levels is considered a promising strategy for AD prevention. 3'-O-acetyl-24-epi-7,8-didehydrocimigenol-3-O- $\beta$-D-xylopryranoside significantly decreased the $A \beta$ production and this effect was accompanied with reduced sAPP $\beta$ production known as a soluble ectodomain APP fragment through $\beta$-secretases in HeLa cells overexpressing amyloid precursor proteins (APPs). This compound also increased the level of SAPP $\alpha$, which is a proteolytic fragment of APP by $\alpha$-secretases. In addition, 3'-O-acetyl24-epi-7,8-didehydrocimigenol-3-O- $\beta$-D-xylopryranoside decreased the protein level of $\beta$-secretases, but the protein levels of A disintegrin and metalloproteinase (ADAM) family, especially ADAM10 and ADAM17, are increased. Thus, 3'-O-acetyl-24-epi7,8-didehydrocimigenol-3-O- $\beta$-D-xylopryranoside could be useful in the development of AD treatment in the aspect of amyloid pathology.
\end{abstract}

Key Words: Alzheimer's disease, Anti-amyloidogenic effect, Secretases

\section{INTRODUCTION}

$A D$ is a progressive neurodegenerative disorder which is recognized as the most common form of dementia among the elderly characterized by progressive dysfunction of cognition and memory (Scheltens et al., 2016). It affects the millions of the elderly and the number of $A D$ patients has dramatically increased. In the worldwide, approximately 35.6 million people are currently affected from $A D$, but it is expected that the number of people living with $A D$ will reach 135 million people in 2050 (Prince et al., 2013; Kim et al., 2015a). Although the cause of $A D$ has not yet been fully understood, but several studies investigated that $A \beta$ deposition is one of the major causes of $A D$ pathology in the early onset familial AD (Hardy and Orr, 2006). Senile plaques formed by extracellular $A \beta$ accumulation are one of the $A D$ pathologic hallmarks which believed to not only interfere the synapses neu- ron communication but also lead to cell death (Karran and De Strooper, 2016). The A $\beta$ is known to be produced from APP (Gu et al., 2018). The shedding of APP generates SAPP $\beta$ and CTF $\beta$ through $\beta$-secretase, and consequential CTF $\beta$ cleavage produces $A \beta 40$ as well as $A \beta 42$ by $\gamma$-secretase (Park, 2010; Prince et al., 2013). However, $\alpha$-secretase cleaves APP and then generates SAPP $\alpha$ and CTF $\alpha$ precluding the $A \beta$ formation (Park, 2010). Eventually, the $A \beta$ production will directly be related with the SAPP $\beta$ generation and contrary correlated with the proportion of sAPP $\alpha$ (Kim et al., 2015b). Considering such previous findings, reduction of $A \beta$ or delaying the $A \beta$ deposition in the brain would be the possible therapeutic targets in the treatment or prevention of AD (Hardy and Orr, 2006; Kim et al., 2015b; Karran and De Strooper, 2016). Furthermore, various secretases would be the key factors to modulate to deliver such beneficial effects. The ADAM family are known as $\alpha$-secretases such as ADAM9, ADAM10, and ADAM17,

\section{Open Access https://doi.org/10.4062/biomolther.2020.195}

This is an Open Access article distributed under the terms of the Creative Commons Attribution Non-Commercial License (http://creativecommons.org/licenses/by-nc/4.0/) which permits unrestricted non-commercial use, distribution, and reproduction in any medium, provided the original work is properly cited.
Received Nov 2, 2020 Revised Dec 16, 2020 Accepted Dec 17, 2020 Published Online Feb 23, 2021

\section{*Corresponding Author}

E-mail: hoyang@sejong.ac.kr

Tel: +82-2-3408-1959, Fax: +82-2-3408-4336

${ }^{\dagger}$ The first two authors contributed equally to this work. 
which catalyze the APP ectodomain shedding. ADAM10 is the major ADAM family member which is responsible for the constitutive activity, while other ADAM family such as ADAM9 and ADAM17 are accountable for the cleavage regulation (Vassar et al., 2009; Kuhn et al., 2010; Kim et al., 2015a).

Several drugs are used for slowing down the progression of $A D$, but they do not significantly delay symptom development or cure the disease. However naturally derived therapeutics showed advantages in slowing down $A D$ development and delaying the onset of symptoms (Kim et al., 2015b; Gu et al., 2018; Chen et al., 2019; Lee et al., 2019). Considering the facts that natural products usually show less side effects compared to the synthetic chemicals and exhibit diversified beneficial effects, natural resources are gaining much attentions in the field of neurodegenerative diseases. Cimicifuga dahurica (Turcz.) Maxim. (C. dahurica) is traditionally used as an antipyretic and analgesic in East Asia region such as Korea, China, Japan, and Russia. Several studies have been conducted to determine the activity of specific flavonoid compounds isolated from C. dahurica. Distinguishable compounds such as cycloartane-type triterpenoids, indolinone alkaloids, phenolics contained in this medicinal plant are believed to have antioxidative effects, anti-cancer, and anti-inflammatory (Tian et al., 2007; Qin et al., 2016; Zhang et al., 2016; Lv et al., 2017; Nguyen Phuong Thao et al., 2018). However, the A $\beta$ inhibition effects of compounds isolated from $C$. dahurica have not been studied. In this study, we hypothesized that a compound isolated from $C$. dahurica roots might exhibit anti-A $\beta$ effects in APP overexpressing Hela cells. To test this hypothesis, we examined the effect of the compound on $A \beta$ production and its underlying mechanisms by investigating the formation of SAPP $\alpha$ and SAPP $\beta$ as well as the activities of both $\alpha$ - and $\beta$-secretases.

\section{MATERIALS AND METHODS}

\section{Chemicals and reagents}

Rabbit anti-APP antibodies to detection the C-terminal of APP were purchased from Sigma-Aldrich Co (St. Louis, MO, USA). FBS was purchased from ATCC Company (Manassas, VA, USA). DMEM, penicillin/streptomycin, G418, and $0.25 \%$ trypsin-EDTA were purchased from GIBCO-BRL Company (Carlsbad, CA, USA). Zeocin were purchased from Invitrogen Company (Carlsbad, CA, USA). Rabbit anti-GAPDH, anti-rabbit horseradish peroxidase linked IgG, anti-ADAM9 antibodies and lysis buffer were obtained from Cell Signaling Technology (Danvers, MA, USA). Anti-BACE1 antibody, and anti-APP antibody to detection both mAPP and imAPP were obtained from Abcam Company (Cambridge, UK). Anti-ADAM10 antibody was obtained from Calbiochem Company (San Diego, CA, USA). Anti-TACE and anti-ADAM17 antibodies were obtained from Chemicon Company (Billerica, MA, USA). All other chemicals were of analytical grade obtained from Sigma-Aldrich Co.

\section{Plant materials}

The roots of $C$. dahurica were obtained from Naemome Dah (Ulsan, Korea) which is the herbal resource company on February 2016. Prof. Young Ho Kim from Chungnam National University (Daejeon, Korea) identified and the herbarium of the College of Pharmacy, Chungnam National University de- posited a voucher specimen (CNU-16003).

\section{Plant extraction and isolation of compounds}

The $95 \%$ ethanol extract of $C$. dahurica $(65.3 \mathrm{~g})$ was prepared by extracting dried roots of $C$. dahurica $(2.5 \mathrm{~kg})$ with ethanol and concentrating. Then, the extract was successively partitioned with n-hexane, $\mathrm{CH}_{2} \mathrm{Cl}_{2}$, and $\mathrm{H}_{2} \mathrm{O}$. From the $\mathrm{CH}_{2} \mathrm{Cl}_{2}$ fraction and water layer, 52 compounds were isolated by several rounds of column chromatography and their structures were elucidated by spectroscopic methods. These results have been previously reported elsewhere (Thao et al., 2017a, 2017b, 2017c). Among the 52 compounds isolated from $C$. dahurica, 3'-O-acetyl-24-epi-7,8-didehydrocimigenol-3-O- $\beta$ D-xylopryranoside (Comp 27) was selected for further studies on its beneficial effects against the cytotoxicity of amyloidogenic Hela cell line.

\section{Cell culture and viability assay}

Hela cells stably transfected with an APP carrying Swedish mutation (APPsw) using BioT (Bioland Scientific LLC, Paramount, CA, USA) according to the manufacturer's instructions. APPsw transfected HeLa cells were grown in DMEM supplemented with $10 \%$ FBS, $1 \%$ penicillin/streptomycin, $260 \mu \mathrm{g} / \mathrm{mL}$ Zeocin and $400 \mu \mathrm{g} / \mathrm{mL} \mathrm{G} 418$. The cells were maintained $37^{\circ} \mathrm{C}$ under a humidified atmosphere of $5 \% \mathrm{CO}_{2}: 95 \%$ air. The stock sample solution was dissolved in DMSO and kept at $-20^{\circ} \mathrm{C}$, and diluted to the final concentration in fresh media before each experiment. The final DMSO concentration of the sample solutions did not exceed $0.5 \%$ in all experiments.

For measuring the cell viability, we used the EZ-Cytox kit according to the manufacturer's instruction obtained from DAEILLAB Co (Cheongwon, Korea). Hela cells were seeded in 96-well plates for $24 \mathrm{~h}$ and then various concentrations of comp $27(0-10 \mu \mathrm{M})$ were treated for $12 \mathrm{~h}$. The microplate reader (BIO-TEK ${ }^{\circledR}$ Dower-Wave; BioTek, Winooski, VT, USA) used to measured absorbance at $450 \mathrm{~nm}$. The results were expressed as the percentage of MTT reduction, assigning the $100 \%$ value to the absorbance of the control group.

\section{Western blot analysis}

APPsw-transfected HeLa cells were collected after they were treated with the indicated concentrations of samples and chemicals for the indicated times. After harvested the cells using PBS ( $\mathrm{pH} 7.2$ ), the proteins from cell pellets were lysed in a cold lysis buffer containing protease inhibitor cocktail obtained from Sigma-Aldrich Co. The lysates were centrifuged at $13,000 \mathrm{rpm}$ for $20 \mathrm{~min}$ at $4^{\circ} \mathrm{C}$. The protein content of the supernatant was determined by the Bradford assay obtained from Bio-Rad Laboratories (Hercules, CA, USA) and used in the subsequent experiments. Protein from each sample was separated by sodium dodecyl sulfate-polyacrylamide gel electrophoresis (SDS-PAGE) and then transferred to polyvinylidene fluoride membranes. After blocked membranes with $5 \%$ non-fat milk for $1 \mathrm{~h}$ at room temperature, the membranes incubated overnight at $4^{\circ} \mathrm{C}$ with primary antibodies including anti-APP, ADAM9, ADAM10, ADAM17, and BACE1. The membranes were washed three times using tris-buffered saline buffer with tween 20 (TBST). The membranes were incubated with horseradish peroxidase-conjugate anti-rabbit IgG antibodies for $1 \mathrm{~h}$. Immunoreactive bounds were visualized using enhanced chemiluminescence (ECL) Advance western blotting detection reagents (34095, Bio-Rad Laboratories). Lu- 
minescent Image Analyzer (LAS-4000, Fujifilm, Minato City, Tokyo, Japan) performed for the Imaging and quantitative densitometry analyses. All the protein levels were normalized to that of GAPDH.

\section{$A \beta$ and sAPPa peptide assay}

APPsw-transfected HeLa cells were cultured with the compound or DMSO in DMEM for $8 \mathrm{~h}$ and then the medium was harvested for subsequent analyses. For the secreted $A \beta$ detection, the kits for $\mathrm{A} \beta 42$ (KHB3442) and $\mathrm{A} \beta 40$ (KHB3482) were obtained from Invitrogen Company and used according to the supplier's instructions. For sAPP $\alpha$ detection, sAPP $\alpha$ (27734) ELISA kit obtained from IBL Company was used in this study according to the supplier's instructions as well.

\section{Statistical analysis}

Data were analyzed with Prism 7.0 software (GraphPad Software Inc., San Diego, CA, USA) using one-way analysis of variance (ANOVA) followed by the Tukey multiple comparison test. Statistical significance was set at $p<0.05$ and the results are expressed as the mean \pm SEM.

\section{RESULTS}

The structure of comp 27 isolated from $C$. dahurica is shown in Fig. 1A. To test the effect of comp 27 on cell viability, HeLa cells transfected with APPsw were treated with comp 27 $(1.25,2.5,5,7.5$, and $10 \mu \mathrm{M})$ for $8 \mathrm{~h}$. It did not affect cell viability except for $10 \mu \mathrm{M}$ concentration (Fig. 1B). Therefore, we used these concentrations except $10 \mu \mathrm{M}$ in this study.

Then, we examined the effect of comp 27 on $A \beta$ secretion. Cells were incubated with $2.5,5$, and $7.5 \mu \mathrm{M}$ comp 27 for $8 \mathrm{~h}$, and we measured the levels of $A \beta 42$ and $A \beta 40$ used specific ELISA kits from the conditioned media. The production of both were decreased in a dose-dependent manner. The A $\beta 42$ level was reduced by $66.7 \%, 56.1 \%$, and $46.2 \%$ at $2.5,5$, and 7.5 $\mu \mathrm{M}$ of comp 27, respectively (Fig. $1 \mathrm{C}$ ). The A $\beta 40$ level was also reduced by $75.7 \%, 64.5 \%, 52.5 \%$ at $2.5,5$, and $7.5 \mu \mathrm{M}$ of comp 27, respectively (Fig. 1D).

$\beta$-Secretase and $\gamma$-secretase generated $A \beta$ through sequential cleavage of APP. On the other hand, $\alpha$-secretase and $\gamma$-secretase generated precluding $A \beta$ by cleavage within the A $\beta$ domain. Thus, we further tested the effects of comp 27 on the production of APP proteolytic fragments, SAPP $\beta$ and SAPP $\alpha$, as well as the APP expressions to investigate the two pathways. The secreted level of SAPP $\alpha$ was increased by $116.2 \%, 121.1 \%$, and $131.2 \%$ at $2.5,5$, and $7.5 \mu \mathrm{M}$ of comp 27 , respectively (Fig. 2A). In addition, treatment of $7.5 \mu \mathrm{M}$ comp 27 significantly decreased the level of sAPP $\beta$ to $50.6 \%$ (Fig. 2B, 2C). On the other hand, comp 27 did not change the levels of both mature and immature APP (mAPP and imAPP) (Fig. 2C, 2D).

Comp 27 increased SAPP $\alpha$ secretion and decreased the secretion of $A \beta$ and sAPP $\beta$. However, it did not affect total APP expression. Therefore, we expected that comp 27 may affect either ADAM family or BACE1 which are respectively acting as $\alpha$ - and $\beta$-secretases.

Next, we investigated whether comp 27 affect ADAM family expressions and activities. Cells were incubated with 2.5,
A

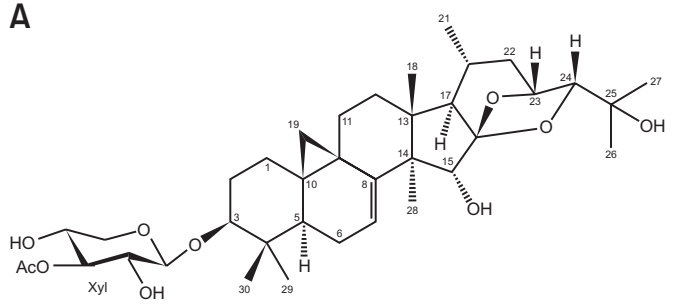

B

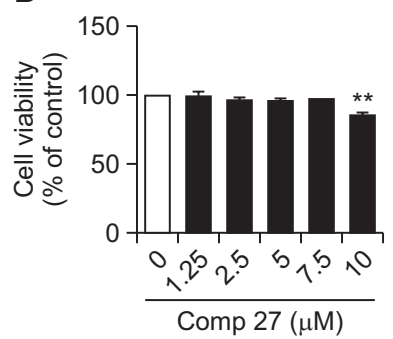

C

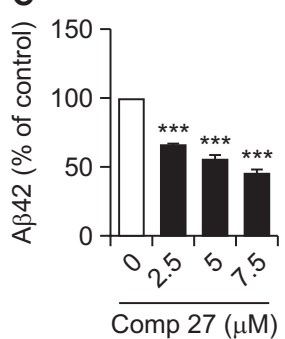

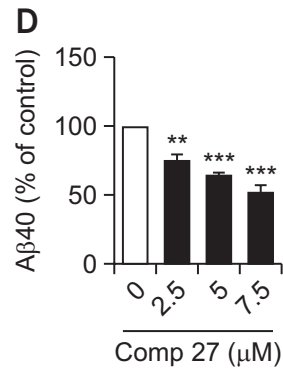

Fig. 1. Effect of comp 27 on cell viability and $A \beta$ secretion. (A) Chemical structure of 3'-O-acetyl-24-epi-7,8-didehydrocimigenol-3-O- $\beta$-Dxylopyranoside (comp 27). (B) The effect of comp 27 on cell viability. (C, D) ELISA result of $A \beta 42$ and $A \beta 40$ secretion after comp 27 treatment. ${ }^{* *} p<0.01,{ }^{* * *} p<0.001$ compared to the control group.

A

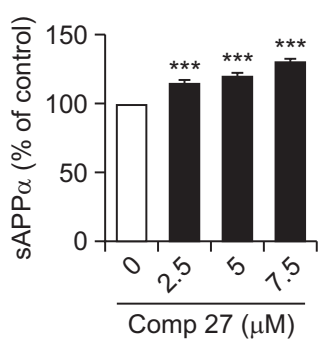

B

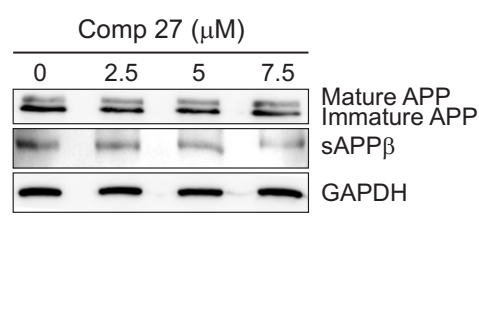

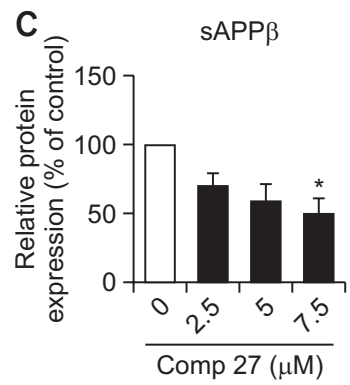

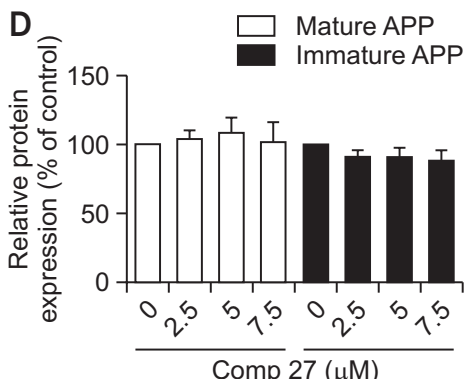

Fig. 2. Effect of comp 27 on APP processing. (A) ELISA result of sAPP $\alpha$ after comp 27 treatment. (B) Western blot analysis of sAPP $\beta$, mature APP and immature APP after comp 27 treatment. (C) Comp 27 dose-dependently decreased the level of sAPP $\beta$. (D) Comp 27 did not change the levels of both mature and immature forms of APP. ${ }^{*} p<0.05,{ }^{* * *} p<0.001$ compared to the control group. 
A

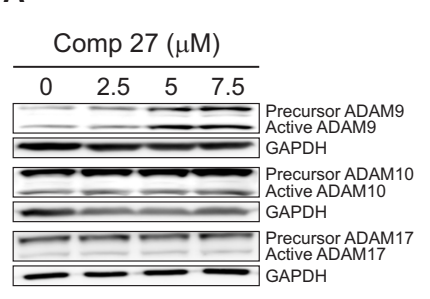

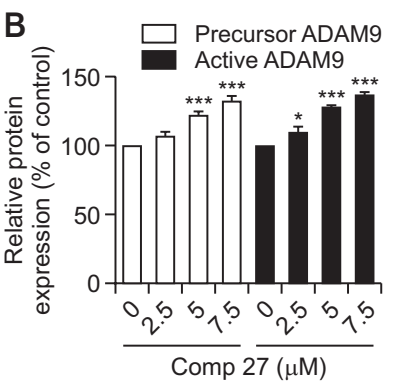

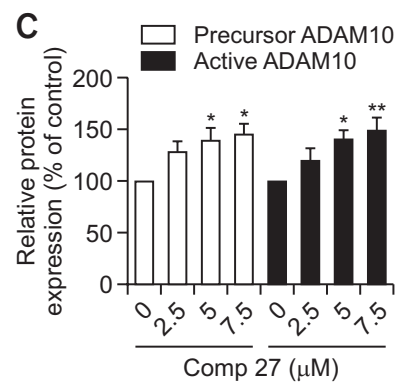

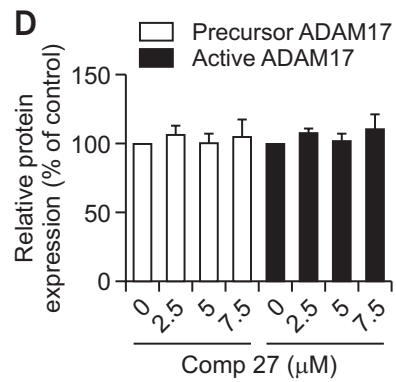

Fig. 3. Effect of comp 27 on the Expression of ADAM family. (A) Western blot analysis of ADAM9, 10 and 17 after comp 27 treatment. (B) Comp 27 dose-dependently increased the levels of precursor and active ADAM9. (C) Comp 27 dose-dependently increased the levels of precursor and active ADAM10. (D) Comp 27 did not change the levels of both precursor and active ADAM17. ${ }^{*} p<0.05$, ${ }^{* *} p<0.01$, and ${ }_{* * *} p<0.001$ compared to the control group.
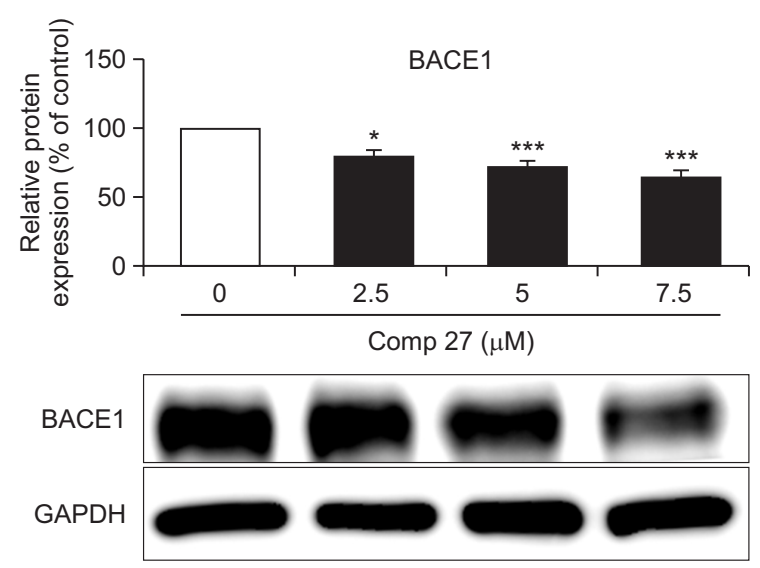

Fig. 4. Effect of comp 27 on BACE1 Expression. comp 27 dosedependently reduced the expression of BACE1. ${ }^{*} p<0.05$ and ${ }_{* * *} p<0.001$ compared to the control group.

5 , and $7.5 \mu \mathrm{M}$ of comp 27 for $8 \mathrm{~h}$, and the levels of ADAMs in lysates were measured using Western blot analysis. ADAM9 and ADAM10 exist as a pro-enzyme state, which are converted to mature form to cleavage of the APP (Lammich et al., 1999). We found that both precursor and active ADAM9 and ADAM10 were increased in a dose-dependent manner (Fig. 3A). Treatment with $7.5 \mu \mathrm{M}$ of the compound increased ADAM9 levels to $136.5 \%$, and ADAM10 levels to $150.3 \%$, respectively (Fig. 3B, 3C). On the other hand, comp 27 did not change the expression of any forms of ADAM17, precursor and active ADAM17 (Fig. 3D).

We tried to determine whether comp 27 influences BACE1 protein expression. As we expected, compound 27 dose-dependently decreased BACE1 expression. The level of BACE1 was decreased by $77.2 \%, 68.5 \%$, and $55.5 \%$ at $2.5,5$, and 7.5 $\mu \mathrm{M}$ comp 27, respectively (Fig. 4).

\section{DISCUSSION}

Cimicifuga dahurica (Turcz.) Maxim. is commonly called 'shengma'. It is one of the ancient herbal medicines that has been subject of extensive studies. It is distributed widely in Northeast Asia and Russia, and traditionally used as an anti- pyretic and analgesic agent (Thao et al., 2018). In this study, we first showed that comp 27, one of the isolated compounds from C. dahurica, significantly decreased both $A \beta 42$ and $A \beta 40$ secretion in HeLa cells overexpressing mutant APPs within the range of no cytotoxicity. We further investigated the possible underlying mechanism of comp 27. Comp 27 increases sAPP $\alpha$ secretion which may be attributed to the expression of $\alpha$-secretases. As expected, comp 27 increased ADAM9 and ADAM10 expression without affecting ADAM 17 activity. This result indicates that the comp 27 -induced $A \beta$ reduction occurs through increasing the level of $\alpha$-secretase, especially ADAM9 and ADAM10. In addition, we found that comp 27 decreases SAPP $\beta$ formation concomitantly. Reduction of secreted sAPP $\beta$ might be due to either decreasing APP expression itself or inhibiting the expression level of BACE1, which is a $\beta$-secretase responsible for the cleavage generating the $A \beta$ peptides in the amyloidogenic pathology. According to our observation, comp 27 did not affect the expressions of both mature and immature APPs. Instead, we detected that comp 27 decreased BACE1 expression which can be the main cause of reduced expression of sAPP $\beta$. BACE1 expression, however, can be regulated at the amount of transcription, translation, or protein degradation. So, further studies need to be performed to investigate the specific mechanisms underlying the comp 27 in such processes.

$A \beta$ oligomers stimulate the kind of biological signaling pathway involving oxidative stress and neuroinflammation (Agostinho et al., 2010). This process leads not only a neuronal synapses and dendrites impairment but also disintegration of the neural circuits and neuronal loss eventually (Vargas et al., 2018). Accordingly, reducing the $A \beta$ generation using any substance is considered to likely a good approach for treatment or prevention of AD. To date, it was not successful to develop an effective drug to stop or modify the progression of AD. Natural products could be an excellent source to reveal a hint for the therapeutic candidates against chronic and complexed disorders including neurodegenerative diseases. Since APP is cleaved within its extracellular domain by $\alpha$-secretase or $\beta$-secretase, the promoting effect of comp 27 on $\alpha$-secretase expression could decrease amyloidogenic process of APP by $\beta$-secretase. It also suggests that inhibitory effect of comp 27 on $\beta$-secretase expression could result in the same effect. Thus, our data suggest that comp 27 decreases $A \beta$ production in vitro via modulation of two kinds of enzymes directly involved in APP cleavage. Confirmation of such significant anti- 
$A \beta$ effects in the future animal studies would promote comp 27 to be a possible therapeutic candidate for the AD pathology.

\section{CONFLICT OF INTEREST}

The authors declare no competing financial interest.

\section{ACKNOWLEDGMENTS}

This work was funded by the National Research Foundation of the Ministry of Science, Information and Communications Technology (ICT) \& Future Planning of Republic of Korea (NRF-2015M3A9A5030735) and the Bio-Synergy Research Project (NRF-2012M3A9C4048793).

\section{REFERENCES}

Agostinho, P., Cunha, R. A. and Oliveira, C. (2010) Neuroinflammation, oxidative stress and the pathogenesis of Alzheimer's disease. Curr. Pharm. Des. 16, 2766-2778.

Chen, X., Xu, B., Nie, L., He, K., Zhou, L., Huang, X., Spencer, P., Yang, X. and Liu, J. (2019) Flavanol-rich lychee fruit extract substantially reduces progressive cognitive and molecular deficits in a triple-transgenic animal model of Alzheimer disease. Nutr. Neurosci. doi: 10.1080/1028415X.2019.1673527 [Online ahead of print].

Gu, M. Y., Chun, Y. S., Zhao, D., Ryu, S. Y. and Yang, H. O. (2018) Glycyrrhiza uralensis and semilicoisoflavone $B$ reduce $A \beta$ secretion by increasing PPAR $\gamma$ expression and inhibiting STAT3 phosphorylation to inhibit BACE1 expression. Mol. Nutr. Food. Res. 62, e1700633.

Hardy, J. and Orr, H. (2006) The genetics of neurodegenerative diseases. J. Neurochem. 97, 1690-1699.

Karran, E. and De Strooper, B. (2016) The amyloid cascade hypothesis: are we poised for success or failure? J. Neurochem. 139 Suppl 2, 237-252.

Kim, J., Park, Y., Chun, Y. S., Cha, J. W., Kwon, H. C., Oh, M. S., Chung, S. and Yang, H. O. (2015a) Effect of lycoris chejuensis and its active components on experimental models of Alzheimer's disease. J. Agric. Food Chem. 63, 6979-6988.

Kim, J. M., Hwang, K. W., Joo, H. B. and Park, S. Y. (2015b) Antiamyloidogenic properties of dryopteris crassirhizoma roots in Alzheimer's disease cellular model. J. Food Biochem. 39, 478-484.

Kuhn, P. H., Wang, H., Dislich, B., Colombo, A., Zeitschel, U., Ellwart, J. W., Kremmer, E., Rossner, S. and Lichtenthaler, S. F. (2010) ADAM10 is the physiologically relevant, constitutive alpha-secretase of the amyloid precursor protein in primary neurons. EMBO J. 29, 3020-3032.

Lammich, S., Kojro, E., Postina, R., Gilbert, S., Pfeiffer, R., Jasionowski, M., Haass, C. and Fahrenholz, F. (1999) Constitutive and regulated alpha-secretase cleavage of Alzheimer's amyloid precursor protein by a disintegrin metalloprotease. Proc. Natl. Acad. Sci.
U.S.A. 96, 3922-3927.

Lee, J., Cho, E., Kwon, H., Jeon, J., Jung, C. J., Moon, M., Jun, M., Lee, Y. C., Kim, D. H. and Jung, J. W. (2019) The fruit of Crataegus pinnatifida ameliorates memory deficits in beta-amyloid proteininduced Alzheimer's disease mouse model. J. Ethnopharmacol. 243, 112107.

Lv, C., Yang, F., Qin, R., Qi, Z., Zhou, W. and Lu, J. (2017) Bioactivityguided isolation of chemical constituents against $\mathrm{H} 2 \mathrm{O} 2$-induced neurotoxicity on PC12 from Cimicifuga dahurica (Turcz.) Maxim. Bioorg. Med. Chem. Lett. 27, 3305-3309.

Park, S. Y. (2010) Potential therapeutic agents against Alzheimer's disease from natural sources. Arch. Pharm. Res. 33, 1589-1609.

Prince, M., Bryce, R., Albanese, E., Wimo, A., Ribeiro, W. and Ferri, C. P. (2013) The global prevalence of dementia: a systematic review and metaanalysis. Alzheimers Dement. 9, 63-75.e2.

Qin, R., Zhao, Y., Zhao, Y., Zhou, W., Lv, C. and Lu, J. (2016) Polyphenolic compounds with antioxidant potential and neuro-protective effect from Cimicifuga dahurica (Turcz.) Maxim. Fitoterapia 115, 52-56.

Scheltens, P., Blennow, K., Breteler, M. M., de Strooper, B., Frisoni, G. B., Salloway, S. and Van der Flier, W. M. (2016) Alzheimer's disease. Lancet 388, 505-517.

Thao, N. P., Kim, J. H., Thuy Luyen, B. T., Dat, N. T. and Kim, Y. H. (2017a) In silico investigation of cycloartane triterpene derivatives from Cimicifuga dahurica (Turcz.) Maxim. roots for the development of potent soluble epoxide hydrolase inhibitors. Int. J. Biol. Macromol. 98, 526-534.

Thao, N. P., Lee, Y. S., Luyen, B. T. T., Oanh, H. V., Ali, I., Arooj, M., Koh, Y. S., Yang, S. Y. and Kim, Y. H. (2018) Chemicals from Cimicifuga dahurica and their inhibitory effects on pro-inflammatory cytokine production by LPS-stimulated bone marrow-derived dendritic cells. Nat. Prod. Sci. 24, 194-198.

Thao, N. P., Luyen, B. T., Lee, J. S., Kim, J. H. and Kim, Y. H. (2017b) Soluble epoxide hydrolase inhibitors of indolinone alkaloids and phenolic derivatives from Cimicifuga dahurica (Turcz.) Maxim. Bioorg. Med. Chem. Lett. 27, 1874-1879.

Thao, N. P., Luyen, B. T. T., Lee, J. S., Kim, J. H., Dat, N. T. and Kim, Y. H. (2017c) Inhibition potential of cycloartane-type glycosides from the roots of cimicifuga dahurica against soluble epoxide hydrolase. J. Nat. Prod. 80, 1867-1875.

Tian, Z., Si, J., Chang, Q., Zhou, L., Chen, S., Xiao, P. and Wu, E. (2007) Antitumor activity and mechanisms of action of total glycosides from aerial part of Cimicifuga dahurica targeted against hepatoma. BMC Cancer 7, 237.

Vargas, L. M., Cerpa, W., Munoz, F. J., Zanlungo, S. and Alvarez, A. R. (2018) Amyloid-beta oligomers synaptotoxicity: the emerging role of EphA4/c-Abl signaling in Alzheimer's disease. Biochim. Biophys. Acta Mol. Basis Dis. 1864, 1148-1159.

Vassar, R., Kovacs, D. M., Yan, R. and Wong, P. C. (2009) The betasecretase enzyme BACE in health and Alzheimer's disease: regulation, cell biology, function, and therapeutic potential. J. Neurosci. 29, 12787-12794.

Zhang, L. L., Si, J. Y., Zhang, L. J., Xiao-Wei, H., Lin, L., Li, R. Y., Chen, D. and Cao, L. (2016) Synergistic anti-tumor activity and mechanisms of total glycosides from Cimicifuga dahurica in combination with cisplatin. Chin. J. Integr. Med. doi: 10.1007/s11655-015-21083 [Online ahead of print]. 